\title{
Characterization of U-Pu-Zr and U-Pu-Mo Fuel Alloys in Transmission Electron Microscope
}

\author{
A. Aitkaliyeva, B. Miller, T. P. O’Holleran, J. R. Kennedy, B. H. Sencer, T. Hyde \\ Idaho National Laboratory, Idaho Falls, USA
}

Uranium-plutonium-zirconium (U-Pu-Zr) and uranium-plutonium-molybdenum (U-Pu-Mo) fuels, known for their high burnup and good thermal response, have been considered as candidate fuels for advanced fast reactors. During their lifetime in the reactor, irradiation in combination with high temperatures can result in swelling of the fuel and its interaction with the cladding. As a result of the complex fuel-cladding chemical interaction (FCCI), integrity of fuel and cladding could be compromised and therefore should be comprehensively examined. As part of the fuel cycle research and development (FCRD) program, formation of intermetallic phases within fuel-cladding interaction zones was investigated in scanning electron microscope (SEM) and transmission electron microscope (TEM).

The diffusion couples were assembled from U-23.3Pu-8.7Zr and U-23.2Pu-9.1Mo fuel ingots and Fe-12Cr cladding. Prior to assembling, the surface of fuel slugs and cladding were polished to mirror polish. Each fuel slug was placed in contact with the cladding material and compressed in a jig, lined with tantalum foil on the inside to prevent an unwanted reaction between diffusion couples and the holder. After assembling, diffusion couples were placed in a furnace and annealed at $750^{\circ} \mathrm{C}$ for 75 hours under continuous flow of argon. Immediately after annealing the jig was quenched in water to preserve the phases formed upon heat treatment.

Diffusion couples were examined using scanning/transmission electron microscopy (STEM/TEM), selected area diffraction (SAD), and X-ray energy dispersive spectroscopy (XEDS) techniques. Cross-sectional specimens were prepared in dual beam focused ion beam (FIB)/scanning electron microscope using a lift-out approach. Prior to TEM specimen preparation, the surface of diffusion couples was examined with energy and wavelength dispersive spectroscopy (WDS) techniques in scanning electron microscope to determine the exact location of the formed interaction layer and to examine resulting microstructure and intermetallic phases. Numerous lift-outs were performed within interaction zone to ensure that all phases formed upon annealing were analyzed.

Transmission electron micrographs of the intermetallic phases formed in both $\mathrm{Zr}$ and Mo fuels and the corresponding elemental X-ray maps are provided in figures 1-2. Elemental X-ray mapping was accompanied by the quantitative XEDS analysis and crystal structure examination. Refer to figure 3 for the overview of intermetallic phases formed in Mo-based fuel. The results of the extensive TEM analysis will be provided in the paper. The discussion on phase evolution will be based on existing equilibrium phase diagram and phase-segregation mechanisms. 


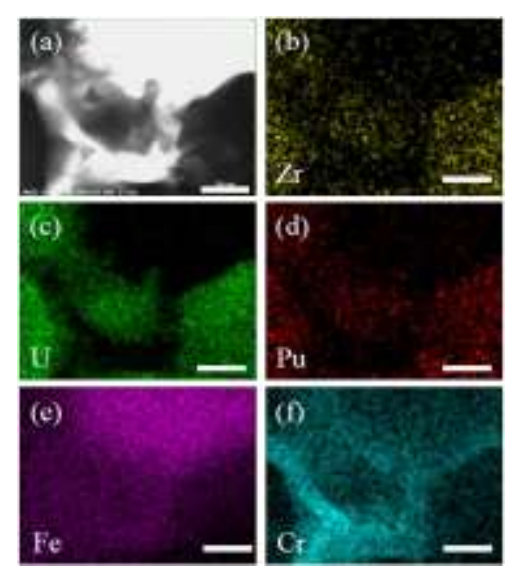

Figure 1. (a) Transmission electron micrograph of the intermetallic phase formed between U-Pu$\mathrm{Zr}$ fuel and Fe-12 Cr cladding. (b)-(f) XEDS maps of the elements, acquired within region shown in (a). The scale bar denotes $2 \mu \mathrm{m}$.
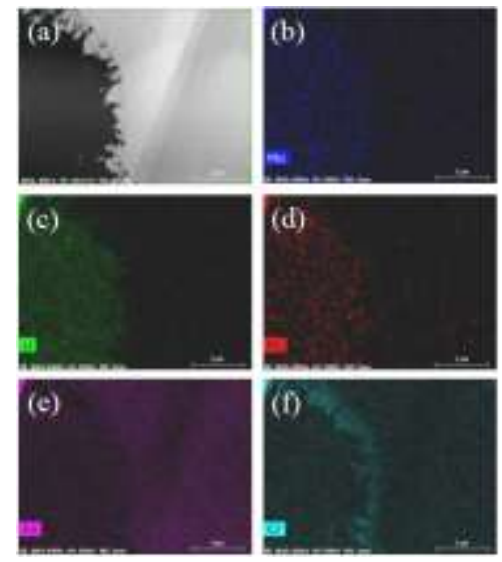

Figure 2. (a) Transmission electron micrograph of the intermetallic phase formed between U-PuMo fuel and Fe-12 Cr cladding. (b)-(f) XEDS maps of the elements, acquired within region shown in (a). The scale bar denotes $2 \mu \mathrm{m}$.

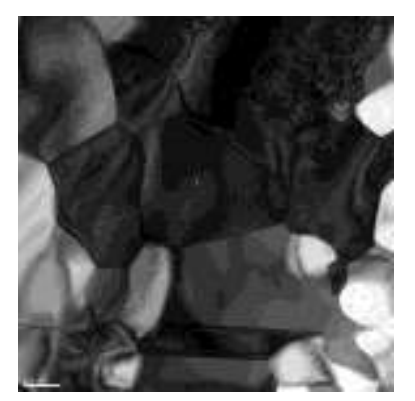

Figure 3. Bright-field transmission electron micrograph of the intermetallic phases formed between U-Pu-Mo fuel and Fe-12 Cr cladding. The scale bar denotes $0.2 \mu \mathrm{m}$.

[1] This work is supported by the US Department of Energy through Fuel Cycle Research and Development (FCRD) program at INL. 